\title{
Functional characterization of gibberellin signaling-related genes in Panax ginseng
}

\author{
Jinsoo Kim - Woo-Ri Shin - Yang-Hoon Kim • Donghwan Shim • Hojin Ryu
}

Received: 14 August 2021 / Revised: 17 September 2021 / Accepted: 17 September 2021

(C) Korean Society for Plant Biotechnology

\begin{abstract}
Gibberellins (GAs) are essential phytohormones for plant growth that influence developmental processes and crop yields. Recent functional genomic analyses of model plants have yielded good characterizations of the canonical GA signaling pathways and related genes. Although Panax ginseng has long been considered to have economic and medicinal importance, functional genomic studies of the GA signaling pathways in this crucial perennial herb plant have been rarely conducted. Here, we identified and performed functional analysis of the GA signaling-related genes, including PgGID1s, PgSLY1s, and PgRGAs. We confirmed that the physiological role of GA signaling components in $P$. ginseng was evolutionarily conserved. In addition, the important functional domains and amino acid residues for protein interactions among active GA, GID1, $\mathrm{SCF}^{\mathrm{SLY} 1}$, and RGA were also functionally conserved. Prediction and comparison of crystallographic structural similarities between PgGID1s and AtGID1a supported their function as GA receptors. Moreover, the subcellular localization and GAdependent promotion of DELLA degradation in $P$. ginseng was similar to the canonical GA signaling pathways in other plants. Finally, we found that overexpression of PgRGA2 and PgSLY1-1 was sufficient to complement the GA-related phenotypes of atgid la/c double- and rga quintuple-mutants, respectively. This critical information for these GA signaling
\end{abstract}

J. Kim $\cdot$ H. Ryu $(\bowtie)$

Department of Biology, Chungbuk National University,

Cheongju 28644, Republic of Korea

e-mail: hjryu96@chungbuk.ac.kr

W.-R. Shin · Y.-H. Kim • H. Ryu

Department of Biological Sciences and Biotechnology, Chungbuk National University, Cheongju 28644, Republic of Korea

D. Shim $(\bowtie)$

Department of Biological Sciences, Chungnam National University, Daejeon 34134, Republic of Korea

e-mail: dshim104@cnu.ac.kr genes has the potential to facilitate future genetic engineering and breeding of $P$. ginseng for increased crop yield and production of useful substances.

Keywords Panax ginseng, GA, Hormone, Signal transduction, Genome engineering

\section{Introduction}

Gibberellins (GA) are plant hormones in the tetracyclic diterpenoid family and play a critical role in diverse plant growth and development. Gibberellins are involved in seed germination, flowering, pollen maturation, stress tolerance and secondary growth (Olszewski et al. 2002; Reid 1993; Ross et al. 1997). Recent studies have revealed that there is a complicated signaling interplays between gibberellins and the internal signaling of various hormonal pathways (auxin, abscisic acid, jasmonic acid, ethylene, etc.) and external biotic and abiotic factors. In particular, these signaling crosstalks were found to be critical for plant growth and development and stress tolerance (Achard and Genschik 2009; Bari and Jones 2009; Harberd et al. 2009). As a major negative regulator of GA signaling, DELLA is primarily involved in the regulation of plant development and stress tolerance. Recent studies have shown that inactivation of DELLA not only affects plant growth and development, but also reduces tolerance to cold and salt stress and increases susceptibility to pathogens. A variety of internal and external signaling pathways applied to plants directly influence the synthesis, metabolism and signal transduction of gibberellins and ultimately regulate the function of the DELLA protein (Sun 2011).

In the presence of active GA signaling cues, GA is recognized by the nuclear-localized receptor protein GID1 (GIBBERELLIN INSENSITIVE DWARF 1), and the GA/GID1 complex induces rapid degradation of DELLA 
proteins (Harberd et al. 2009; Sun 2010). There are a total of five DELLA genes in the genome of Arabidopsis (RGA (REPRESSOR OF GA1-3), GAI (GIBBERELLIC ACID INSENSITIVE), RGA-LIKE1 (RGL1), RGL2 and RGL3), showing spatio-temporal expression patterns for each stage of plant development (Cheng et al. 2004; Dill and Sun 2001; King et al. 2001; Lee et al. 2002; Tyler et al. 2004). This indicates that the development and growth of various plants regulated by gibberellin is regulated by tissue specific modulations. It is known that the degradation of DELLA is facilitated by the interaction of GA/GID1 complex with the F-box protein SLEEPY1 (SLY1) (Griffiths et al. 2006; McGinnis et al. 2003; Sasaki et al. 2003; Ueguchi-Tanaka et al. 2005). The DELLA domain does not act in interactions with the F-box protein but is essential for GAinduced proteolysis to occur (Dill et al. 2001; Dill et al. 2004). GID1 is essential for direct binding to DELLA, LEXLE and VHYNP motifs conserved within the domains of DELLA proteins in a GA-dependent manner. Protein structure studies confirmed that GID1 contains a carboxyterminal core domain that forms a GA-binding pocket and an amino-terminal extension domain (N-Ex) that serves as a cap for the pocket (Sun 2011). Although there is no direct interaction between DELLA and GA, DELLA functions in stabilization of the GA-GID1-DELLA complex. In the presence of GA, DELLA is recognized by the $\mathrm{SCF}^{\mathrm{SLY} 1}$ ubiquitin E3 ligase complex for polyubiquitination and subsequent degradation by the 26S proteasome (Dill et al. 2004; Fu et al. 2002; Sasaki et al. 2003).

Korean ginseng (Panax ginseng C.A. Meyer) is one of the perennial medicinal herb plants and has been used as an important medicinal plant for thousands of years ( $\mathrm{Hu}$ 1976;). Recently, the pharmaceutical compounds with high utility have been discovered in the various ginseng tissues including fruit, leaves and stems, and they are used importantly in health functional foods, pharmaceuticals, and cosmetics (Jang et al. 2014; Seo et al. 2015; Yasukawa et al. 2016). With the recently developed PacBio single-molecule real-time (SMRT) Iso-seq analysis, we successfully provided to more accurate information of the full-length transcriptome sequences of $P$. ginseng (Jo et al. 2017; Hong et al. 2018). Moreover, the eight PgGIDl genes were identified, and GA was identified as an important factor that can increase crop yields by promoting secondary growth of the storage main roots of $P$. ginseng (Hong et al. 2021). However, the structural underlying mechanisms of the PgGID1 receptors and downstream pathways including DELLA and SCF ${ }^{\text {SLY1 }}$ have not been reported yet. In this study, the tertiary structure of PgGID1s was predicted and their role as GA receptors was investigated. In addition, five PgRGA genes and PgSLY1 gene were identified and characterized as their functions as GA signaling components in $P$. ginseng.

\section{Materials and Methods}

PgGID1 protein structure modeling and docking with $\mathrm{GA}_{3}$

The crystallographic structure of Arabidopsis thaliana GID1a (PDB : 2ZSH) was retrieved from the Protein Data Bank (https://www.rcsb.org/). This structure was used to a template to predict the core domain of AtGID1a, PgGID1A, PgGID1C and PgGID1D, respectively, using the PHYRE II analysis and PyMOL structure visualization software. These include separation of the co-crystallized ligand and protein, as well as addition of the hydrogens to the protein, which are omitted in the protein databank files. Protein was calculated for structural inspection and docking using MOE 2016.0802 with the following set-up: force-field was Amber10:EHT, termination was $0.001 \mathrm{kcal} / \mathrm{mol}$.

Phylogenetic analysis and protein sequence alignments

The protein sequences of RGA- and SLY1 (GID2)-related genes were selected from previous studies (Hong et al. 2021; Jo et al. 2017). A phylogenetic tree based on protein sequence alignment was generated using MEGA version 7.0 software by the neighbor-joining method with a bootstrap value of 1000 (Kumar et al. 2016). An online program, SMS (http;//www. Bioinformatics.org), was applied to align the protein sequence. Percentage of sequences that must agree for identity or similarity coloring to be added was $70 \%$.

\section{Protoplast transient expression assay}

The full-length cDNAs of PgRGAs and PgSLY1-1 (Hong et al. 2021) were cloned into plant expression vectors containing GFP sequence tags in the $\mathrm{C}$ terminus driven by the $35 S:: C 4 P P D K$ promoter as previously described (Lee et al. 2021). For protoplasts transient expression assays, about $4 \times 10^{4}$ protoplasts were transfected with $20 \mu \mathrm{g}$ of plasmid DNA and then incubated under constant light condition at $23^{\circ} \mathrm{C}$ for $6 \mathrm{~h}$. For the subcellular localization, GFP-tagged constructs were transfected into protoplasts. GFP and chloroplast auto fluorescence were observed with a fluorescence microscope (Nikon). 
Plant materials and growth conditions

Arabidopsis thaliana ecotype Col-0 and Ler were used as wild-type controls and protoplast isolation. atgidla/c knockout (Col-0) and rga quintuple (Ler) mutants were used as the genetic backgrounds of transgenic lines. Arabidopsis seeds were germinated on solid media $(\mathrm{pH} 5.7$ - 5.8) containing 1/2 Gamborg B5 medium (Duchefa, Netherlands), $1 \%$ sucrose and $0.8 \%$ plant-agar. All plants were grown in a greenhouse at $20 \sim 22{ }^{\circ} \mathrm{C}$ under a long-day conditions (16-h light/8-h dark cycles) with $60 \%$ humidity.

Transgenic plants and immunoblotting assay

To generate transgenic plants overexpressing HA-tagged Pgsly1-d (Genbank \# MZ621175) and PgRGA2 in the atgidla/c and rga quintuple mutant background, respectively, cDNA fragment was cloned into $p C B 302 E S$ containing the $35 S$ promoter and $H A$ tag sequences as described previously (Lee et al. 2021). All transgenic lines were generated by Agrobacterium-mediated floral dipping methods. Transgene expression was verified by immunoblotting. Total proteins from seedlings were extracted with protein extraction buffer (50 mM Tris- $\mathrm{HCl}$ ( $\mathrm{pH} 7.5), 75 \mathrm{mM} \mathrm{NaCl}$, $5 \mathrm{mM}$ EDTA, $1 \mathrm{mM}$ dithiothreitol, $1 \times$ protease inhibitor cocktail (Roche), and 1\% Triton X-100). Total protein (3 20 $\mu \mathrm{g})$ was separated by SDS-PAGE (10\% polyacrylamide), transferred to a polyvinylidene difluoride membrane and immunodetected using 1/2,000 dilution of a peroxidaseconjugated high-affinity anti-HA antibody (Roche).

\section{Results and Discussion}

Docking results of the $\mathrm{GA}_{3}$ interacting with the PgGID1 proteins

To confirm the molecular basis of gibberellin receptor (PgGID1s) in P. ginseng (Hong et al. 2021), we initially analyzed the protein sequence and protein 3D structure prediction. For comparison of the possible structural similarity, a docking process was performed in MOE 2016.08, using a bioactive $\mathrm{GA}_{3}$ molecule. The docking pose for optimized $\mathrm{GA}_{3}$ with PgGID1 protein was observed to be situated at similar position for the $\mathrm{GA}_{3}$ molecule crystallized within $2 \mathrm{ZSH}$. The binding mode of $\mathrm{GA}_{3}$ with the coredomain of AtGID1A (A), PgGID1A (B), PgGID1C (C) and PgGID1D (D) were depicted in Figure 1. The PgGID1 structure is found to be similar to the AtGID1A from
Arabidopsis thaliana (2ZSH), the core domain of which superimposes with PgGID1 protein. The PgGID1A structure shows a Root Mean Square Deviation (RMSD) of 0.113 $\AA$ to the closest structure 2ZSH (PgGID1C and PgGID1D structure RMSD of $0.144 \AA$ to $2 \mathrm{ZSH}$ ). Using MOE docking simulation, $\mathrm{GA}_{3}$ was docked to each structure. The lowest energy, the docking almost the same as the corresponding X-ray structure (Fig. 1E, F and G). The $\mathrm{GA}_{3}$ molecules are responded the pocket by forming multiple hydrogen bond with Ser residue (AtGID1 - Ser 116 and Ser 191; PgGID1 - Ser 115 and Ser 190)1. The $\mathrm{GA}_{3}$ also formed a salt bridge with AtGID1AArg244 and PgGID1DArg243, whereas in PgGID1AArg243 and PgGID1CArg245 a formed hydrogen bond. These results indicate that the PgGID1s can structurally bind with an active $\mathrm{GA}_{3}$, suggesting that they can act as receptors. Based on these results with our previous study (Hong et al. 2021), the evolutionary conserved GID1 proteins in $P$. ginseng are sufficiently performed as GA signaling receptors and stimulate downstream signaling pathways for the diverse physiological responses.

Identification of $\mathrm{SCF}^{\mathrm{SLY} 1} \mathrm{E} 3$ ubiquitin ligases in $P$. ginseng

We then determined whether the GA signaling related genes are evolutionarily conserved in $P$. ginseng genome. To identify GA signaling components, two genomic data obtained from our previous research (Jo et al. 2017) and other two groups (Waminal et al. 2018; Xu et al. 2017) were analyzed. By a blast analysis based on the amino acid sequence of the GA signaling factor of Arabidopsis, it was possible to obtain 14 genes including $4 S L Y 1$ s and $10 R G A \mathrm{~s}$ in the genome of $P$. ginseng. Each selected gene was named PgSLY1-1 1-4 and PgRGA1-10, and the relationship with those of Arabidopsis and rice GA signaling components was determined through phylogenetic analysis (Fig. 2 and 4). Among the identified genes, we tried to determine whether the F-box protein PgSLY1s have an evolutionarily conserved function for GA signaling pathways. In phylogenetic analysis with SLY1 (GID2) of Arabidopsis and rice, it was confirmed that the relationship between AtSLY1 and these genes was closely related (Fig. 2A). For comparative analysis of protein sequence, we identified the major domains present in the PgSLY1-1 protein (Fig. 2B; Nelson and Steber 2018). We found that the F-box domain to which SKP1 is bound and the GGF/LSL domain important for interaction with DELLA are well conserved (Hirano et al. 2007; Nelson and Steber 2018). We also confirmed that the PgSLY1 protein sequences were similar to the AtSLY1 and OsSLY1 protein sequences (Fig. 2C). 

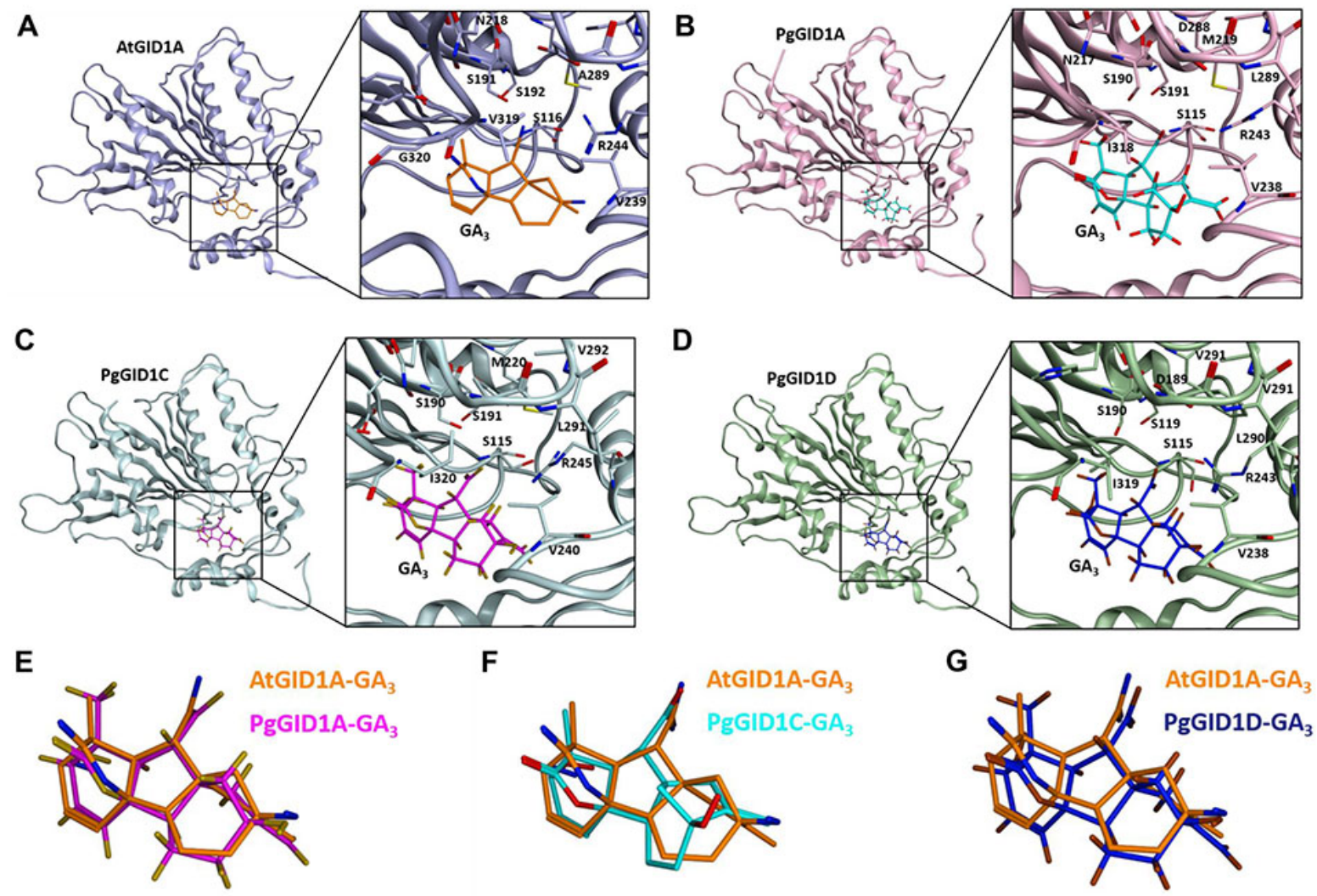

$\mathbf{F}$
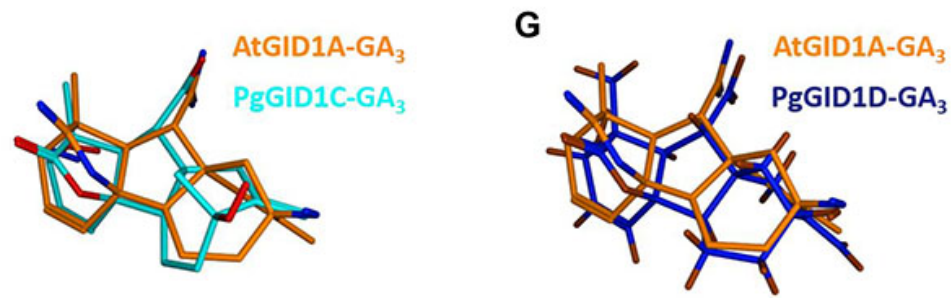

Fig. 1 Molecular docking results of $\mathrm{GA}_{3}$ and GID1 proteins in core domain (HGGS). (A) A model based on GA bound in the AtGID1A structure (PDB ID: 2ZSH). (B-D) Docking results of $\mathrm{GA}_{3}$ in the core domain of AtGID1A and PgGID1A, C and D. (E) Overlay of the docking pose of PgGID1A-GA 3 and the crystallographic pose of AtGID1A-GA 3 in 2ZSH. (F) Overlay of the docking pose of PgGID1C-GA 3 and the crystallographic pose of AtGID1A-GA 3 in 2ZSH. (G) Overlay of the docking pose of PgGID1D-GA and the crystallographic pose of AtGID1A-GA 3 in $2 \mathrm{ZSH}$.

A

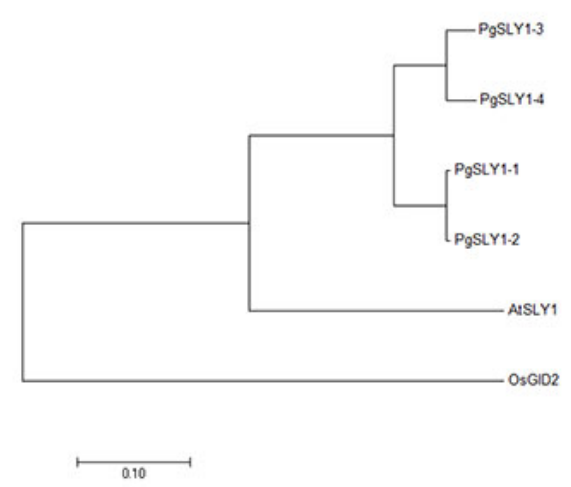

B

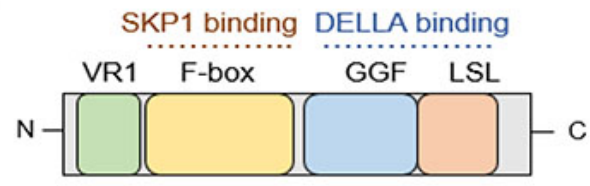

C

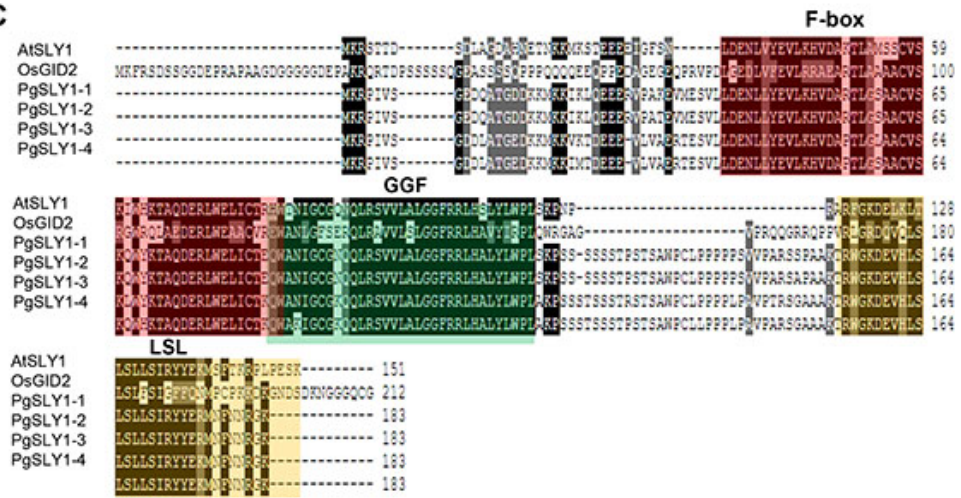

Fig. 2 Phylogenetic analysis and alignment of the amino acid sequences of PgSLY1 proteins (A) Phylogenetic analysis of PgSLY1-1, PgSLY1-2, PgSLY1-3, and PgSLY1-4 proteins and their homologs, including AtSLY1 and OsGID2. The phylogenetic tree was constructed using the MEGA7 program. The horizontal branch lengths are proportional to the estimated number of amino acid substitutions per residue. Bootstrap values were obtained by 1,000 bootstrap replicates. Pg, Panax ginseng; At, Arabidopsis thaliana; Os, Oryza sativa. (B) A topology diagram based on the predicted secondary structure of the PgSLY1-1 protein. (C) Amino acid sequences of SLY1-like proteins from Arabidopsis, rice, and P. ginseng were aligned using SMS (http://www. Bioinformatics.org) 
A



B

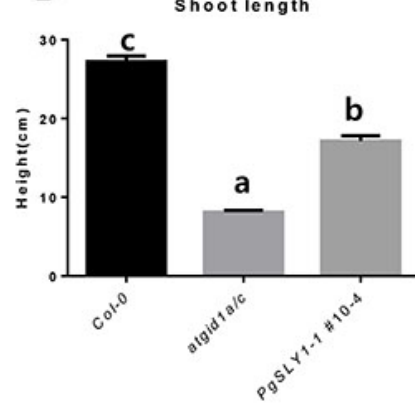

C

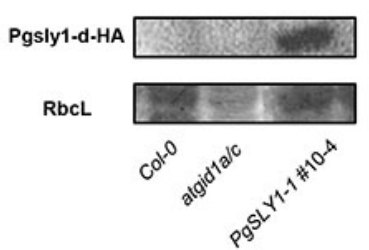

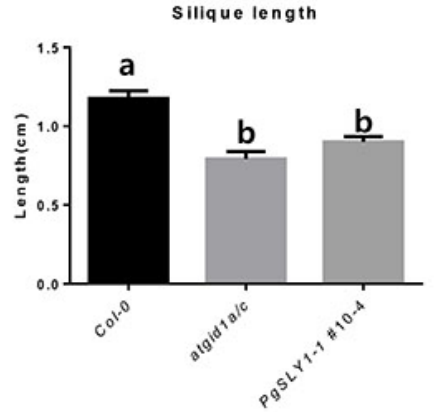

D

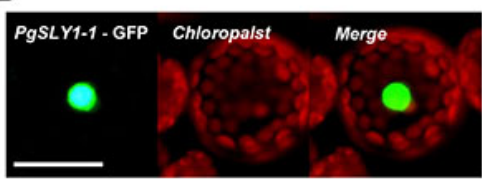

Fig. 3 Functional characterization of PgSLY1-1 in the atgidla/c loss-of-function mutant. (A) Plants overexpressing the $P$. ginseng SLY1-1 gene were rescued the GA-insensitive dwarf phenotypes in shoot (above) and silique (below) of the atgidla/c mutant. Scale bar $=5 \mathrm{~cm}$ (above), $0.5 \mathrm{~cm}$ (below). (B) Measurement of the lengths of the shoots and siliques shown in (A). Error bars represent the standard error $(n>12)$. Different lowercase letters indicate statistically significant differences $(P<0.05$; one-way ANOVA, followed by Tukey's multiple-range test). (C) Western blot analysis of proteins extracted from wild-type, atgid1a/c, and PgSLY1-1overexpressing atgidla/c plants. (D) Nuclear localization of PgSLY1-1 proteins in Arabidopsis protoplasts

The important domains for the functions in GA signaling pathways presented in Fig. $2 \mathrm{~b}$ are also evolutionarily well conserved with high similarity in the amino acid sequences (Fig. 2C).

Overexpression of the nuclear localized PgSLY1-1 partially complemented atgidla/c mutant phenotypes

To figure out whether PgSLY1 has a biological function for GA-related responses in plants, we checked whether the GA-defective dwarf phenotypes were restored by overexpressing it in semi-dwarf atgidla/c mutant plants (Fig. $3 \mathrm{~A})$. Although the phenotype of the stem length was not as high as that of the wild type, it was confirmed that the height of the shoots expressing 35S-PgSLY1-1-HA was partially recovered compared to the GA-defective atgidla/c mutants (Fig. 3B). However, the recovery pattern in the size of the silique of the PgSLY1-1 overexpressing plants was not significant. To further confirm whether this phenotypic recovery of the atgidla/c mutant is due to the overexpression of the PgSLY1-1 gene, the protein expression level was determined (Fig. 3C). These results indicate that the PgSLY1-1 can restore some physiological function in plants with weakened gibberellin signaling. Previous studies have shown that SLY1 is mainly expressed in the nucleus of plant cells (Ariizumi et al. 2011). To confirm the intracellular localization of PgSLY1-1, the GFP-tagged gene was expressed and observed in protoplasts. As present in Fig. 3D, the PgSLY1-1 was localized in the nucleus. Taken together, these results suggest that nuclear-localized PgSLY1-1 acts as an F-box E3 ubiquitin ligase and induces degradation of DELLA protein, an inhibitor of GA signaling.

Identification of DELLA inhibitor proteins and functional analysis in $P$. ginseng

Our findings suggest that the core GA signaling components are functionally conserved in the genome of P. ginseng. Therefore, we tried to check whether the DELLA (RGA) gene, which is a gibberellin signaling inhibitor, can actively play a role as a major negative regulator of GA signaling. As a result of phylogenetic analysis based on the RGA protein sequences of Arabidopsis and rice, it was confirmed that AtRGA1 and 2 are closely related to PgRGAs (Fig. 4A). For comparative analysis based on the amino acid sequence, we identified the major domains present in the PgRGA proteins (Fig. 4B; Nelson and Steber 2018). The DELLA protein has a DELLA regulatory domain and a GRAS functional domain. The DELLA domain at the N-terminus contains a DELLA/TVHYNP motif, which is known to play an important role in binding to GID1 (Nelson and Steber 2018). It is known that a GRAS domain exists at the C-terminus and LHR, VHIID, PFYRE, and SAW sites also confirmed (Nelson and Steber 2018). The VHIID/LHR2 

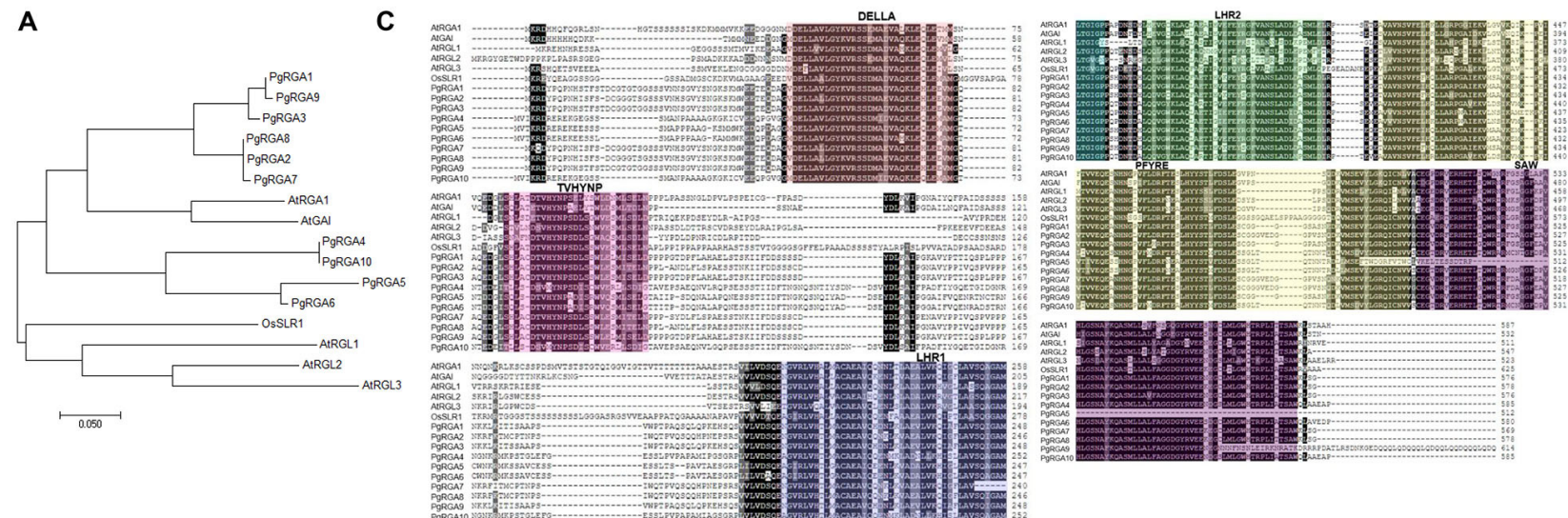

B
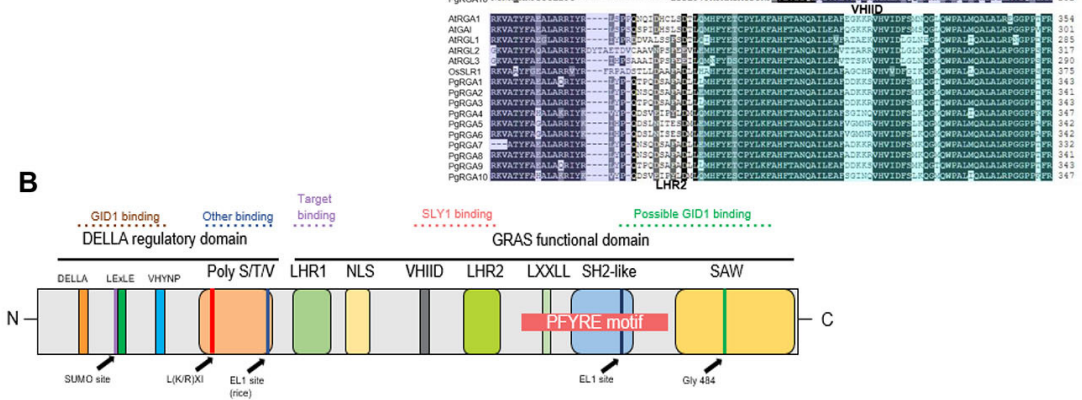

Fig. 4 Identification of DELLA proteins in P. ginseng genome. (A) Phylogenetic analysis of PgRGA1-10 proteins with homologs. The phylogenetic tree was constructed using the MEGA7 program. The horizontal branch lengths are proportional to the estimated number of amino acid substitutions per residue. Bootstrap values were obtained by 1,000 bootstrap replicates. Pg, Panax ginseng; At, Arabidopsis thaliana; Os, Oryza sativa. (B) A topology diagram based on the predicted secondary structure of PgRGA proteins. (C) Amino acid sequences of RGA-like proteins from Arabidopsis, rice, and P. ginseng were aligned using SMS (http://www. Bioinformatics.org)

motif is important for binding to the $\mathrm{SCF}^{\mathrm{SLY} 1}$, and the PFYRE/SAW motif is known to play an important role in inhibiting plant growth. Through protein sequence alignment, it was confirmed that PgRGA had high similarity to the AtRGA and OsRGA1 with conservation of all functional domains (Fig. 4C).

To confirm whether PgRGA performs a biological function in plants, it was observed whether the phenotype was recovered by overexpressing PgRGA2 in Arabidopsis, in which all five $R G A$ s were knocked out (rga quintuple mutant). The rga quintuple plant showed a long hypocotyl and shoot growth phenotypes (Fig. 5A). The PgRGA2 overexpressing $r g a$ quintuple mutant showed a normal growth rate similar to that of the wild type (Fig. 5A). In addition, it was confirmed that the length of the hypocotyl was shortened and restored to a length similar to that of the wild type (Fig. 5A-C). To determine whether the phenotypic recovery of the mutant was due to overexpression of the PgRGA2 gene, the protein expression level was determined (Fig. 5D). These results show that the PgRGA2 identified in $P$. ginseng can replace function in plants in which all five $R G A$ genes are knocked out. Based on these results, we confirmed that these PgRGAs conserved in P. ginseng can act as DELLA, a GA signaling inhibitors in plants. In order to confirm the subcellular localization of PgRGAs, the PgRGA genes tagged with GFP were expressed and observed in the protoplasts (Fig. 5E). We confirmed that all PgRGA proteins were expressed in the nucleus, and these results suggested that the PgRGA could act as an inhibitor of GA signaling in the nucleus, similar to other orthologs. Finally, our results suggest that the GID1 and $\mathrm{SCF}^{\mathrm{SLY} 1}$ of $P$. ginseng can be interacted with the DELLA proteins in the nucleus to promote proteolysis of them. To confirm this, we tested whether the degradation of DELLA proteins was promoted by exogenous GA treatments in the PgRGA2 overexpressing plants (Fig. 5F). We found that the RGA2 protein level was not change significantly in the mock treatments, but the amount of RGA2 was rapidly decreased in the presence of an active $\mathrm{GA}_{3}$. These results suggest that GA signaling initiated from GID1 in $P$. ginseng ultimately induces degradation of DELLA proteins via $\mathrm{SCF}^{\mathrm{PgSLY} 1}$ mediated 26s proteasome pathways.

Through this study, we identified major genes involved in GA signaling known to promote the secondary growth of ginseng storage roots (Hong et al. 2021). As ginseng is cultivated for at least 6 years, it is required to induce 

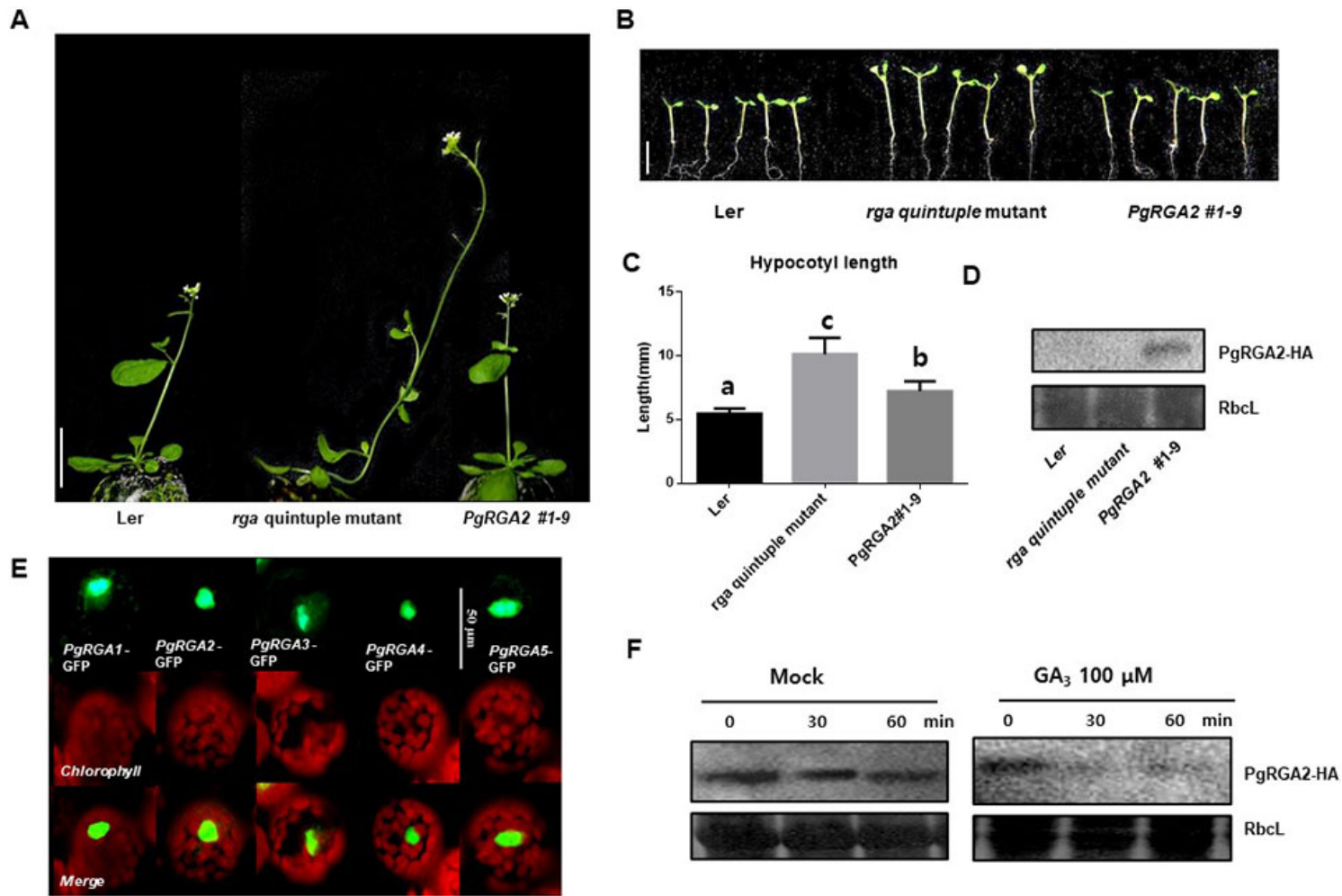

Fig. 5 Functional characterization of $P g R G A 2$ in plants. (A) Plants overexpressing the $P$ gRGA2 gene were rescued the phenotype of the rga quintuple knockout mutants. Scale bar $=3 \mathrm{~cm}$. $(\mathrm{B}, \mathrm{C})$ We measured the hypocotyl lengths of the phenotypes. Scale bar $=0.5 \mathrm{~cm}$. Error bars represent the standard error $(n=10)$. Different lowercase letters indicate statistically significant differences $(P$ $<0.05$; one-way ANOVA, followed by Tukey's multiple-range test). (D) Western blot analysis of proteins extracted from wild-type, rga quintuple-knockout mutant, and PgRGA2-overexpressing rga quintuple-knockout plants. (E) Nuclear localization of PgRGA1-5 proteins in Arabidopsis protoplasts. (F) Exogenous $\mathrm{GA}_{3}$ facilitated PgRGA2 protein degradation. PgRGA2-HA proteins were extracted from the PgRGA2-overexpressing rga quintuple-knockout plants treated with or without $100 \mu \mathrm{m}$ of $\mathrm{GA}_{3}$ for the indicated time in the presence of cycloheximide

the growth of storage roots and the accumulation of various medicinal substances. Most studies for understanding the growth and development of plants have performed using the annual herbaceous model plant system such as Arabidopsis and rice. However, the recently reported study of GA signaling involved in the storage root development of ginseng provided important clues for developing tools promoting the secondary growth of $P$. ginseng, a perennial root crop. The information on the GA signaling genes of $P$. ginseng provided through this study will be used importantly in the development of biotechnology for breeding and increasing the production of useful substances of $P$. ginseng.

\section{Conflict of Interest Disclosures}

All Authors have read the manuscript and declared that they have no conflict of interest.

\section{Acknowledgments}

This research was supported by Chungbuk National University Korea National University Development Project (2020)

\section{References}

Achard P, Genschik P (2009) Releasing the brakes of plant growth: how GAs shutdown DELLA proteins. Journal of experimental botany 60(4):1085-1092

Ariizumi T, Lawrence PK, Steber CM (2011) The role of two F-box proteins, SLEEPY1 and SNEEZY, in Arabidopsis gibberellin signaling. Plant physiology 155(2):765-775

Bari R, Jones JD (2009) Role of plant hormones in plant defence responses. Plant molecular biology 69(4):473-488

Cheng H, Qin L, Lee S, Fu X, Richards DE, Cao D, Luo D, Harberd NP, Peng J (2004) Gibberellin regulates Arabidopsis floral development via suppression of DELLA protein function. Development 131(5):1055-1064 
Dill A, Jung H-S, Sun T-p (2001) The DELLA motif is essential for gibberellin-induced degradation of RGA. Proceedings of the National Academy of Sciences 98(24):14162-14167

Dill A, Sun T-p (2001) Synergistic derepression of gibberellin signaling by removing RGA and GAI function in Arabidopsis thaliana. Genetics 159(2):777-785

Dill A, Thomas SG, Hu J, Steber CM, Sun T-p (2004) The Arabidopsis F-box protein SLEEPY1 targets gibberellin signaling repressors for gibberellin-induced degradation. The Plant Cell 16(6):1392-1405

Fu X, Richards DE, Ait-Ali T, Hynes LW, Ougham H, Peng J, Harberd NP (2002) Gibberellin-mediated proteasome-dependent degradation of the barley DELLA protein SLN1 repressor. The Plant Cell 14(12):3191-3200

Griffiths J, Murase K, Rieu I, Zentella R, Zhang Z-L, Powers SJ, Gong F, Phillips AL, Hedden P, Sun T-p (2006) Genetic characterization and functional analysis of the GID1 gibberellin receptors in Arabidopsis. The Plant Cell 18(12):3399-3414

Harberd NP, Belfield E, Yasumura Y (2009) The angiosperm gibberellin-GID1-DELLA growth regulatory mechanism: how an "inhibitor of an inhibitor" enables flexible response to fluctuating environments. The Plant Cell 21(5):1328-1339

Hirano K, Nakajima M, Asano K, Nishiyama T, Sakakibara H, Kojima M, Katoh E, Xiang H, Tanahashi T, Hasebe M (2007) The GID1-mediated gibberellin perception mechanism is conserved in the lycophyte Selaginella moellendorffii but not in the bryophyte Physcomitrella patens. The Plant Cell 19 (10):3058-3079

Hong CP, Kim J, Lee J, Yoo S-i, Bae W, Geem KR, Yu J, Jang I-b, Jo IH, Cho H, Shim D, Ryu H (2021) Gibberellin signaling promotes the secondary growth of storaage roots in Panax ginseng. International Journal of Molecular Science 22:8694

Hong J, Kim H, Ryu H (2018) Identification of ABSCISIC ACID (ABA) signaling related genes in Panax ginseng. J Plant Biotechnology (45):306-314

Hu SY (1976) The genusPanax (ginseng) in Chinese medicine. Economic Botany 30(1):11-28

Jang H-J, Han I-H, Kim Y-J, Yamabe N, Lee D, Hwang GS, Oh M, Choi K-C, Kim S-N, Ham J (2014) Anticarcinogenic effects of products of heat-processed ginsenoside $\mathrm{Re}$, a major constituent of ginseng berry, on human gastric cancer cells. Journal of agricultural and food chemistry 62(13):2830-2836

Jo I-H, Lee J, Hong C, Lee D, Bae W, Park S-G, Ahn Y, Kim Y, Kim J, Lee J (2017) Isoform sequencing provides a more comprehensive view of the panax ginseng transcriptome. Genes 8(9):228

King KE, Moritz T, Harberd NP (2001) Gibberellins are not required for normal stem growth in Arabidopsis thaliana in the absence of GAI and RGA. Genetics 159(2):767-776

Kumar S, Stecher G, Tamura K (2016) MEGA7: molecular evolutionary genetics analysis version 7.0 for bigger datasets. Molecular biology and evolution 33(7):1870-1874

Lee J, Kim H, Park SG, Hwang H, Yoo Si, Bae W, Kim E, Kim J, Lee HY, Heo TY (2021) Brassinosteroid-BZR1/2-WAT1 module determines the high level of auxin signalling in vascular cambium during wood formation. New Phytologist 230(4):1503-1516

Lee S, Cheng H, King KE, Wang W, He Y, Hussain A, Lo J, Harberd NP, Peng J (2002) Gibberellin regulates Arabidopsis seed germination via RGL2, a GAI/RGA-like gene whose expression is up-regulated following imbibition. Genes \& development 16(5):646-658

McGinnis KM, Thomas SG, Soule JD, Strader LC, Zale JM, Sun T-p, Steber CM (2003) The Arabidopsis SLEEPY1 gene encodes a putative F-box subunit of an SCF E3 ubiquitin ligase. The Plant Cell 15(5):1120-1130

Nelson SK, Steber CM (2018) Gibberellin hormone signal perception: down-regulating DELLA repressors of plant growth and development. Annual Plant Reviews online:153-187

Olszewski N, Sun T-p, Gubler F (2002) Gibberellin signaling: biosynthesis, catabolism, and response pathways. The Plant Cell 14 (suppl 1):S61-S80

Reid JB (1993) Plant hormone mutants. Journal of Plant Growth Regulation 12(4):207-226

Ross JJ, Murfet IC, Reid JB (1997) Gibberellin mutants. Physiologia Plantarum 100(3):550-560

Sasaki A, Itoh H, Gomi K, Ueguchi-Tanaka M, Ishiyama K, Kobayashi M, Jeong D-H, An G, Kitano H, Ashikari M (2003) Accumulation of phosphorylated repressor for gibberellin signaling in an F-box mutant. Science 299(5614):1896-1898

Seo E, Kim S, Lee S, Oh B-C, Jun H-S (2015) Ginseng berry extract supplementation improves age-related decline of insulin signaling in mice. Nutrients 7(4):3038-3053

Sun T-p (2010) Gibberellin-GID1-DELLA: a pivotal regulatory module for plant growth and development. Plant physiology 154(2):567-570

Sun T-p (2011) The molecular mechanism and evolution of the GA -GID1-DELLA signaling module in plants. Current Biology 21(9):R338-R345

Tyler L, Thomas SG, Hu J, Dill A, Alonso JM, Ecker JR, Sun T-p (2004) DELLA proteins and gibberellin-regulated seed germination and floral development in Arabidopsis. Plant physiology 135(2):1008-1019

Ueguchi-Tanaka M, Ashikari M, Nakajima M, Itoh H, Katoh E, Kobayashi M, Chow T-y, Yue-ie CH, Kitano H, Yamaguchi I (2005) GIBBERELLIN INSENSITIVE DWARF1 encodes a soluble receptor for gibberellin. Nature 437(7059):693

Waminal NE, Pellerin RJ, Jang W, Kim HH, Yang T-J (2018) Characterization of chromosome-specific microsatellite repeats and telomere repeats based on low coverage whole genome sequence reads in Panax ginseng. Plant Breeding and Biotechnology 6(1):74-81

Xu J, Chu Y, Liao B, Xiao S, Yin Q, Bai R, Su H, Dong L, Li X, Qian J (2017) Panax ginseng genome examination for ginsenoside biosynthesis. Gigascience 6(11):gix093

Yasukawa K, Whang W-K, Ko S-K (2016) Inhibitory effects of ginseng (Panax ginseng) berry on tumour promotion and inflammatory ear oedema induced by TPA. Journal of Nutritional Therapeutics 4(4):143-148 